\title{
THE POSITIVE FIXED POINTS OF BANACH LATTICES
}

\author{
BRUCE CHRISTIANSON
}

(Communicated by William Davis)

\begin{abstract}
Let $Z$ be a Banach lattice endowed with positive cone $C$ and an order-continuous norm $\|$.$\| . Let G$ be a left-amenable semigroup of positive linear endomorphisms of $Z$. Then the positive fixed points $C_{0}$ of $Z$ under $G$ form a lattice cone, and their linear span $Z_{0}$ is a Banach lattice under an order-continuous norm $\|\cdot\|_{0}$ which agrees with $\|$.$\| on C_{0}$. A counterexample shows that under the given conditions $Z_{0}$ need not contain all the fixed points of $Z$ under $G$, and need not be a sublattice of $(Z, C)$. The paper concludes with a discussion of some related results.
\end{abstract}

Let $G$ be a semigroup. We denote by $m(G)$ the Banach space of all bounded linear functions from $G$ into the real numbers $R$, under the supremum norm. We denote by $m^{*}(G)$ the Banach dual of $m(G)$. With each $T \in G$ we associate an endomorphism $T_{m}$ of $m(G)$ defined by

$$
\left(T_{m} b\right)(U)=b(T U) \quad \text { for } U \in G \text { and } b \in m(G)
$$

where $T U$ denotes the composite of $T$ and $U$ under the semigroup operation.

An element $p \in m^{*}(G)$ is called a mean for $G$ iff

$$
\inf _{T \in G} b(T) \leq p(b) \leq \sup _{T \in G} b(T) \text { for all } b \in G
$$

and left-invariant for $G$ iff

$$
T_{m}^{\prime} p=p \text { for all } T \in G,
$$

where $T_{m}^{\prime}$ denotes the adjoint of $T_{m}$.

Following M. Day [1, p. 108] we call the semigroup $G$ left-amenable iff there exists a left-invariant mean for $G$. In particular, any Abelian semigroup is left-amenable [1, Theorem 4, p. 108].

A Banach lattice is said to have order-continuous norm iff every decreasing sequence of positive elements is norm convergent [3, 5.12, p. 92; 5.10(d), p. 89].

Theorem. Let $(Z, C,\|\|$.$) be a Banach lattice with order-continuous norm. Let$ $G$ be a left-amenable semigroup of positive linear operators from $Z$ into $Z$. Define

$$
C_{0}=\{x \in C: T x=x \text { for all } T \in G\}, \quad Z_{0}=C_{0}-C_{0} .
$$

Received by the editors November 3, 1988; and in revised form March 21, 1989. 1980 Mathematics Subject Classification (1985 Revision). Primary 46B30, 52A43. 
Then $\left(Z_{0}, C_{0}\right)$ is a vector lattice. by

Further, let $|\cdot|_{0}$ be the corresponding lattice modulus and define $\|\cdot\|_{0}$ on $Z_{0}$

$$
\|x\|_{0}=\left\||x|_{0}\right\|
$$

Then $\left(Z_{0}, C_{0},\|\cdot\|_{0}\right)$ is a Banach lattice with order-continuous norm.

Proof. We begin by showing that each pair $\{x, y\}$ in $Z_{0}$ possesses a least upper bound in $Z_{0}$. (Note that the cones $C$ and $C_{0}$ induce the same partial ordering on $Z_{0}$, since $C \cap Z_{0}=C_{0}$.) Without loss of generality, suppose that $x$ and $y$ are positive, and let $x \vee y$ denote their lub in $Z$. The set $\{T(x \vee y): T \in G\}$ is bounded above, since

$$
T(x \vee y) \leq T(x+y)=x+y \text { for all } T \in G,
$$

hence this set has a least upper bound $z$ in $Z$ by the order-continuity of the norm [3, 5.10(a), p. 89]. We shall show that $z$ is the required lub in $Z_{0}$ of $x$ and $y$.

We show $z \in C_{0}$. Let $Z^{*}$ denote the Banach dual of $Z$, endowed with dual norm and dual positive cone. Each $T \in G$ is bounded [3, 5.3, p. 84] and so has an adjoint $T^{\prime}$ on $Z^{*}$. Define (for the given $x$ and $y$ ) a positive linear map $j$ from $Z^{*}$ into $m(G)$ by

$$
(j f)(T)=\left(T^{\prime} f\right)(x \vee y) .
$$

Let $p$ be a left-invariant mean for $G$ and set $w=j^{\prime} p \in Z^{* *}$, where $j^{\prime}$ is the adjoint of $j$. Since $j T^{\prime}=T_{m} j$ we have for $T \in G$ that

$$
T^{\prime \prime} w=T^{\prime \prime}\left(j^{\prime} p\right)=j^{\prime}\left(T_{m}^{\prime} p\right)=j^{\prime} p=w .
$$

We show that $w=i z$ where $i$ is the canonical embedding of $Z$ into $Z^{* *}$. For each $T$ we have

$$
x=T x \leq T(x \vee y) \text { and } y=T y \leq T(x \vee y),
$$

so

$$
x \vee y \leq T(x \vee y) \leq z
$$

But

so

$$
\inf _{T \in G} j f(T) \leq p(j f) \leq \sup _{T \in G} j f(T) \quad \text { for all } f \in Z^{*},
$$

$$
\begin{aligned}
f(x \vee y) & \leq \inf _{T \in G} f(T(x \vee y)) \leq w(f) \\
& \leq \sup _{T \in G} f(T(x \vee y)) \leq f(z) \quad \text { for all } f \geq 0 .
\end{aligned}
$$

Hence

$$
i(x \vee y) \leq w \leq i z
$$

Now we have

$$
i T(x \vee y)=T^{\prime \prime} i(x \vee y) \leq T^{\prime \prime} w=w \quad \text { for all } T \in G .
$$


But $i z$ is the lub of $\{i T(x \vee y): T \in G\}$ since $i$ preserves arbitrary suprema by order-continuity [3, p. $89 \mathrm{ff} 5.9 \mathrm{C}]$. Hence $i z \leq w$. We already have $w \leq i z$, so $i z=w$, giving $T z=z$, whence $z \in C_{0}$ as promised.

If $u \in C_{0}$ with $x \vee y \leq u$ then

$$
T(x \vee y) \leq T u=u \text { for all } T \in G
$$

so $z \leq u$. We already have $x \vee y \leq z$, thus $z$ is the lub in $Z_{0}$ of $x$ and $y$. This completes the proof that $\left(Z_{0}, C_{0}\right)$ is a vector lattice.

It remains to show that $\|\cdot\|_{0}$ has the required properties. Clearly it is a lattice norm and agrees with the original norm on $C_{0}$. We show that it is a Banach norm for $Z_{0}$ by an argument borrowed from [2, p. 326]. Suppose $\left(x_{n}\right)$ a sequence in $Z_{0}$ such that $\sum_{n}\left\|x_{n}\right\|_{0}$ converges. Write

$$
x_{n}=y_{n}-z_{n} \text { where } y_{n}, z_{n} \in C_{0} \text { with } y_{n}+z_{n}=\left|x_{n}\right|_{0} .
$$

Then

$$
\left\|y_{n}\right\| \leq\left\|\left|x_{n}\right|_{0}\right\|=\left\|x_{n}\right\|_{0}
$$

so $\sum_{n}\left\|y_{n}\right\|$ converges, whence $\sum_{n} y_{n}$ is (monotone) convergent in the norm $\|$.$\| to some y \in Z$. But $C_{0}$ is closed (since the elements of $G$ are bounded) so $y \in C_{0}$, and the norms agree on $C_{0}$, hence $\sum_{n} y_{n}$ is also monotone convergent to $y$ in $\|\cdot\|_{0}$. Similarly $\sum_{n} z_{n}$ converges to some $z \in C_{0}$ and so $\sum_{n} x_{n}$ converges to $y-z \in Z_{0}$ in $\|\cdot\|_{0}$ which is therefore complete for $Z_{0}$.

The order continuity of $\|\cdot\|_{0}$ is inherited from that of $\|\cdot\|$. For let $\left(x_{n}\right)$ be a monotone decreasing sequence in $C_{0}$, then $\left(x_{n}\right)$ is (monotone) convergent in $\|$.$\| to some x \in C$. But $C_{0}$ is closed in $\|$.$\| so x \in C_{0}$, and the norms agree on $C_{0}$ so $\left(x_{n}\right)$ is also monotone convergent to $x$ in $\|\cdot\|_{0}$.

Corollary. Let $V$ be a Banach space and let $V^{+}$be a norm-closed cone in $V$ such that $V=V^{+}-V^{+}$. Suppose further that the Banach dual $(Z, C,\|\|$.$) of$ $V$, endowed with dual norm and dual positive cone, is a Banach lattice. Let $G$ be a semigroup of bounded positive linear operators from $V$ into $V$ and suppose that $G$ is right-amenable (equivalently that $G^{\prime}=\left\{T^{\prime}: T \in G\right\}$ is left-amenable, where $T^{\prime}$ denotes the adjoint of $T$.) Define

$$
C_{0}=\left\{x \in C: T^{\prime} x=x \text { for all } T^{\prime} \in G^{\prime}\right\}, \quad Z_{0}=C_{0}-C_{0} .
$$

Then $\left(Z_{0}, C_{0}\right)$ is a vector lattice. by

Further, let $|\cdot|_{0}$ be the corresponding lattice modulus and define $\|\cdot\|_{0}$ on $Z_{0}$

$$
\|x\|_{0}=\left\||x|_{0}\right\| \text {. }
$$

Then $\left(Z_{0}, C_{0},\|\cdot\|_{0}\right)$ is a Banach lattice.

Proof. As for the Theorem, but define $j$ from $V$ into $m(G)$ by $j f(T)=$ $(x \vee y)(T f)$ and set $z=j^{\prime} p$ directly. 
Remark. In both the Theorem and the Corollary we could equally well have defined $\|\cdot\|_{0}$ to be the Minkowski functional of $c x\left(S_{0} \cup-S_{0}\right)$ where $c x$ denotes convex hull and

$$
S_{0}=\left\{x \in C_{0}:\|x\|=1\right\} .
$$

The Theorem holds in particular for $Z$ any (abstract or concrete) $L^{p}$ space with $1 \leq p<\infty$, and in this case $Z_{0}$ is also an $L^{p}$ space for the same $p$. The Corollary gives a weaker result for $L^{\infty}$ spaces.

Examples. The following examples show that the Theorem is in some sense the strongest result we can hope for under such general conditions.

Example 1. The conclusion of the Theorem may fail if $G$ is not left-amenable. Consider $R^{5}$ as a Lebesgue space with five atoms, and define $P, Q$ by

$$
\begin{aligned}
& P(v, w, x, y, z)=(v, w, x, y, v+w), \\
& Q(v, w, x, y, z)=(v, w, x, y, x+y) .
\end{aligned}
$$

Let $G$ be the semigroup $\{P, Q\}$, then $C_{0}$ is the cone with square base

$$
\{(s, 1-s, t, 1-t): s, t \in[0,1]\}
$$

so $\left(Z_{0}, C_{0}\right)$ is not a lattice. For example $(1,1,2,0,2)$ and $(1,1,1,1,2)$ are incomparable upper bounds in $Z_{0}$ for $(1,0,1,0,1)$ and $(0,1,1,0,1)$.

Example 2. $Z_{0}$ need not equal the set of all fixed points of $Z$ under $G$, and this latter set need not be a lattice. Consider $R^{2}$ as a Lebesgue space and let $G$ be the semigroup $\left\{T^{n}\right\}$ where $T(x, y)=(2 x+y, x+2 y)$. The fixed points are the line $x+y=0$ and $C_{0}=\{0\}$.

Example 3. $Z_{0}$ need not be a sublattice of $(Z, C)$ and the two norms need not agree on the whole of $Z_{0}$. Consider $R^{3}$ as a Lebesgue space and let $G$ be the semigroup $\left\{T^{n}\right\}$ where $T(x, y, z)=(x, y, x+y)$. The points

$$
a=(1,0,1) \quad b=(0,1,1)
$$

have

$$
\begin{array}{cc}
a \wedge b=(0,0,1) & a \wedge_{0} b=(0,0,0) \\
\|a-b\|=2 & \|a-b\|_{0}=4 .
\end{array}
$$

However we do always have $\|\cdot\| \leq\|\cdot\|_{0}$ on $Z_{0}$.

Example 4. $Z_{0}$ need not be norm closed in $(Z,\|\|$.$) and the two norms need$ not therefore be equivalent on $Z_{0}$. Let $Z$ be the Lebesgue (ie $L^{1}$ ) sum of countably many copies of $R^{3}$ considered as a Lebesgue space, and define $T$ by

$$
(T w)_{k}=\left(x_{k}, y_{k}, x_{k}+y_{k}+\left(1-2^{-k}\right) z_{k}\right)
$$

for $w \in Z$ with $w_{k}=\left(x_{k}, y_{k}, z_{k}\right)$. 
Let $G=\left\{T^{n}\right\}$ and define $v$ by $v_{k}=\left(1 / 2^{k},-1 / 2^{k}, 0\right)$. Then $v$ is in the closure of $Z_{0}$ under $\|\cdot\|$ but is not in $Z_{0}$. This example also satisfies the assertions of Examples 2 and 3.

Related Results. If more is assumed about $Z$ or $G$ then stronger results can be proven, i.e., that $Z_{0}$ includes all the fixed points of $Z$ under $G$ (and hence is norm closed), is a sublattice of $Z$, or is the range of some projection or conditional expectation operator with nice properties. Such results are known in a number of cases, i.e.

(1) if $G$ is mean-ergodic $[3,8.4$, p. $188 ; 11.6$, p. 214]. (In case $G$ is compact in the weak operator topology this is equivalent to amenability $[3,17(\mathrm{a})(\mathrm{c})$, p. 222$]$.

(2) if $G$ is a group [3, s. 10, p. $201 ; 2.6 \mathrm{C}$, p. 60$]$.

(3) if $G$ is (uniformly) equicontinuous [3, p. 184].

(4) if $G$ is an $L^{1}$ space and $G$ is contractive [3, p. 184, p. 193 proof].

In these cases, more can often be said about the geometry of $C_{0}$ as a subcone of $C$, i.e., that there is a bijection between the extreme rays of $C_{0}$ and the minimal ideals (or irreducible components) of $G$ (which correspond to minimal $G$-invariant faces of $C)[3,8.7$, p. $190 ; 8.11$, p. 192].

This kind of refinement is difficult in the more general context of our Theorem since the required structure can be quite intricate, although partial results have been obtained by the author under the more restrictive conditions of the Corollary. Nevertheless, the Theorem by itself sometimes provides sufficient information to be useful.

For example, let $S$ be any set of probability measures such that $S$ is a linearly compact simplex (so that $Z=$ lin span $S$ is a vector lattice) and $S$ is closed under the formation of countable convex combinations. Then by [ 3 , 8.2 , p. 113] or [2, p. 325] $Z$ can be normed as an $L^{1}$ space with $S$ precisely the set of positive elements of unit norm in $Z$ (although the lattice join in $Z$ may not be the usual measure join.)

A special case of this occurs when $S$ is a set of (normalized) states for a physical system. Let $G$ be the (Abelian) semigroup generated by some linear mapping (defined on the cone with base $S$ ) corresponding to an evolution of the system. Then the Theorem says that the stationary states of the system generate a vector lattice, even when the evolution is nonconservative (i.e. noncontractive) irreversible (i.e. noninvertible) nonergodic, nonequicontinuous etc.

In some cases, notably if $S$ is vaguely compact or has the Radon-Nikodym property, and if the evolution operator is appropriately continuous, this is sufficient to show that the stationary states possess unique decompositions into pure phases as barycentres (resultants) of boundary measures on $S_{0}$ $[4, \mathrm{pp} .44,50]$. In other cases, the fact that the stationary states form a simplex is of independent interest. 


\section{REFERENCES}

1. M. M. Day, Normed linear spaces, Third Edition, Ergeb. Math. Grenzgeb. 21 (1973), Springer-Verlag, Berlin.

2. C. M. Edwards and M. A. Gerzon, Monotone convergence in partially ordered vector spaces, Ann. Inst. H. Poincaré Phys. Théor. (A)12 (1970), 323-328.

3. H. H. Schaefer, Banach lattices and positive operators, Grundlehren Math. Wiss. 215 (1974), Springer-Verlag, Berlin.

4. G. Winkler, Choquet order and simplices with applications in probabilistic models, Lecture Notes in Math. no. 1145 (1985), Springer-Verlag, Berlin.

School of Information Sciences, Hatfield Polytechnic, Herts, England, Europe.

GB AL10 9AB 